\title{
Verification of Microarchitectural Refinements in Rule-based Systems
}

\author{
Nirav Dave*, Michael Katelman ${ }^{\dagger}$, Myron King*, Arvind*, José Meseguer ${ }^{\dagger}$ \\ * Massachusetts Institute of Technology - Computer Science and Artificial Intelligence Laboratory \\ Cambridge, MA 02139, U.S.A. \\ \{ndave, mdk, arvind\}@csail.mit.edu \\ $\dagger$ University of Illinois at Urbana-Champaign - Department of Computer Science \\ Urbana, IL 61801, U.S.A. \\ \{katelman, meseguer\}@uiuc.edu
}

\begin{abstract}
Microarchitectural refinements are often required to meet performance, area, or timing constraints when designing complex digital systems. While refinements are often straightforward to implement, it is difficult to formally specify the conditions of correctness for those which change cycle-level timing. As a result, in the later stages of design only those changes are considered that do not affect timing and whose verification can be automated using tools for checking FSM equivalence. This excludes an essential class of microarchitectural changes, such as the insertion of a register in a long combinational path to meet timing. A design methodology based on guarded atomic actions, or rules, offers an opportunity to raise the notion of correctness to a more abstract level. In rule-based systems, many useful refinements can be expressed simply by breaking a single rule into smaller rules which execute the original operation in multiple steps. Since the smaller rule executions can be interleaved with other rules, the verification task is to determine that no new behaviors have been introduced. We formalize this notion of correctness and present a tool based on SMT solvers that can automatically prove that a refinement is correct, or provide concrete information as to why it is not correct. With this tool, a larger class of refinements at all stages of the design process can be verified easily. We demonstrate the use of our tool in proving the correctness of the refinement of a processor pipeline from four stages to five.
\end{abstract}

\section{INTRODUCTION}

Modular refinement is an important technique in designing complex digital systems because it eases architectural exploration for better performance, area, and power. For modular refinement to be viable it should be relatively easy to determine if a local change preserves the overall correctness of the design. Generally, it is extremely difficult for a designer to give a full formal correctness specification for a system. Specifying correctness requires a level of knowledge of the overall system and familiarity with formal verification methods that few designers possess. As a consequence, common practice is to settle for partial verification via testing. Testing works, but as test suites tend to be built in conjunction with the design itself, designers rarely gain sufficient confidence in their refinements' correctness until the final stages of the design cycle.

An alternative is to restrict the types of refinements to ones whose local correctness guarantees that the overall behavior will remain unaffected, and designs usually rely on the notion of equivalence supported by the design language semantics for proving or testing local equivalence. As most hardware description languages describe synthesizable systems at the level of gates and wires, this amounts to FSM (finite-state machine) equivalence. Tools usually require the designer to specify the mapping of state elements (e.g., flip-flops), and thus reduce the problem of FSM equivalence to combinational equivalence, which can be performed efficiently. FSMequivalence-preserving refinements have proven to be quite useful because tools are available to prove the local correctness automatically and there is no negative impact on the overall verification strategy. However, FSM refinement is too restrictive, disallowing many desirable changes such as adding a buffer to cut a critical path in a pipeline. Thus these tools are limited to verification in the later stages of design when the timing has been decided.

Recently, languages like Bluespec [4], which describe designs not as gates and wires but as a set of guarded atomic actions (or rules) on state elements, have been proposed. Over the last six years, it has been established that Bluespec programs not only can produce no-compromise hardware [1], but that keeping programs at the rule level allows more flexibility in design and refinements [8], [9]. For instance, the addition of a pipeline stage can be implemented in a natural way by splitting the rule corresponding to the appropriate stage into multiple rules, and introducing state to hold the intermediate results.

A Bluespec program can be reasoned about at two levels. At the first level we deal with rules in an unscheduled manner. The semantics state that we compute by selecting any valid rule (i.e., one whose guard evaluates to true) for execution, update the state by executing the body of the rule, and then repeat the process. This means that the program is naturally non-deterministic, and programs at this level are meant to be correct for all possible traces of execution. At the second level the compiler adds a scheduler which is responsible for resolving the non-determinism so that we may synthesize the program into a high-quality FSM implementation. The choice of scheduler is a purely performance-based concern and should not affect the correctness. We exploit this separation of concerns and focus on the equivalence of systems before scheduling. 


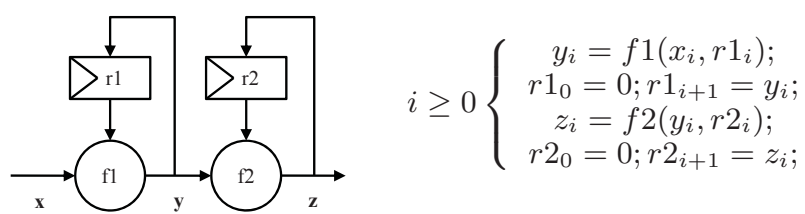

Fig. 1. Initial FSM

Despite the guarded atomic action formalism's deep relation to term-rewriting systems and formal proofs, little work has been done to verify rule-based programs at anything beyond the implementation level. The main contribution of this paper is to define a notion of program equivalence between rulebased programs and describe an SMT-based algorithm that automatically verifies the correctness of "rule splitting" refinements. Our notion of program equivalence is based on the transitive closure of permitted transitions and not on more standard trace-based characterizations. If needed, the distinctions drawn by traced-based characterizations can be expressed programmatically and verified using our notion of equivalence.

A tool based on this algorithm is able to prove the correctness of interesting refinements in a matter of minutes, well within range to be useful as a debugging aid for the designer. We use the tool to show the correctness of several examples including the refinement of a four-stage processor pipeline into a five-stage pipeline.

Paper Organization: In Section II, we discuss the kinds of refinements we want to make and why their correctness cannot be formulated at the FSM level. We also discuss the challenge of verification at the level of rules and discuss how nondeterministic specifications affect the verification task. In Section III, we formalize a notion of equivalence in the context of rule refinements. In Section IV, we discuss the algorithm used by our tool to mechanically verify equivalence using an SMT solver. In Section V, we discuss the verification of a processor program. In the last two sections we discuss related work and present our conclusion.

\section{Motivating Refinement Example}

To understand the challenges of refinement in rule-based systems we must first understand how such refinements differ from refinements of FSMs, motivating our notion of behavior and explaining where the new method and tool are needed.

\section{A. Refining an FSM}

Consider the hardware represented by the FSM system shown in Figure 1. The system consists of two registers $r 1$ and $r 2$, both initially zero, and some combinational logic implementing functions $f 1$ and $f 2$. The critical path in this system goes from $r 1$ to $r 2$ via $f 1$ and $f 2$. In order to improve performance, a designer may want to break this path by adding a buffer (say, a one element FIFO) on the critical path as shown in Figure 2. Though we have not shown the circuitry to do so, we will assume that $r 2$ does not change and the output $z$ is not defined when the FIFO is empty.

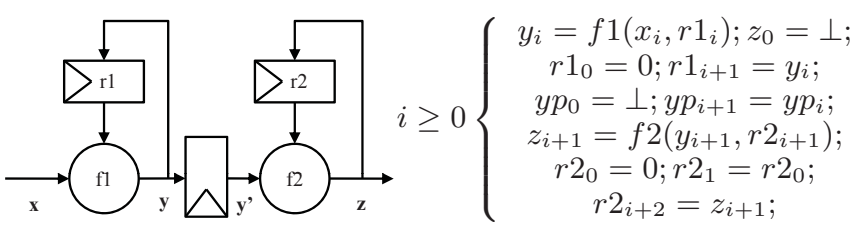

Fig. 2. Refined FSM

In this refined system, the operation that was done in one cycle is now done in two; $f 1$ is evaluated in the first cycle, and $f 2$ in the second. The computation is fully pipelined so that each stage is always productive (except the first cycle of the second stage, when the FIFO buffer is empty) and we have the same cycle-level computation rate. However the clock period in the refined system can be much shorter, thereby increasing system throughput. Though the cycle-by-cycle state of the two FSMs do not match directly, a little bit of analysis will show that the sequence of values assumed by $r 2$ and $z$ are the same in both systems. In other words, the refined system produces the same answer as the original system but one cycle later. Therefore, in many situations such a refinement may be considered correct even though the FSMs of the two systems are not equivalent.

The problem here is that if we don't rely on FSM equivalence then how should we define equivalence? A solution could be to introduce the notion of a message or valid input and output and then define equivalence in terms of input-output sequences of messages as opposed to cycle-by-cycle behavior of input and output. Unless we carry this notion of validity everywhere in the design it is difficult to reason in these terms mechanically.

In the following section we discuss a rule-based description of this example and show how refinements are expressed in such systems.

\section{B. Refinements at the Rule Level}

When designers specify systems using rules, they often have in their mind a particular datapath and FSM, although the exact datapath and FSM is generated by the compiler. For example, a designer may express the FSM design in Figure 1 using a single rule as shown in Figure 3. In contrast to the FSM interface, a rule-based system has no strict notion of clock cycle to determine when data is passed. To deal with this we have added two FIFOs inQ and outQ; when we "take input" we dequeue a value from in $\mathrm{Q}$ and when we have a new value to output we enqueue into out $Q$.

If we assume that a rule executes in one clock cycle then the rule in Figure 3 specifies that every cycle $r 1$ and $r 2$ should be updated, one value should be dequeued from ine, and one value should be enqueued in the outQ. (The approximate logic generated by each rule is shown as a cloud in all the figures; we have omitted the control logic to avoid clutter.) The sequences of values for $r 1$ and $r 2$ match exactly with those in Figure 1. Similarly, the values on the wires $x$ and $z$ 

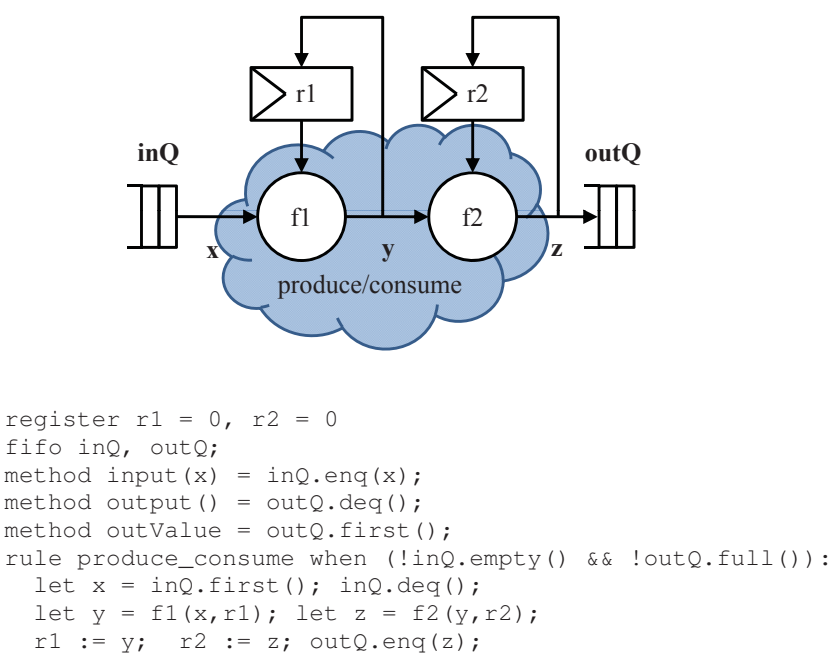

Fig. 3. A Rule-based Specification of the Initial Program

which serve as input and output in the original program match the sequence of values in ing and outQ (see Figure 4).

$$
\begin{array}{ll}
\left(\left[x_{0}, x_{1}, x_{2}, \ldots\right], r 1_{0}, r 2_{0},[]\right) \longrightarrow & \text { where: } r 1_{0}=0 ; r 2_{0}=0 ; \\
\left(\left[x_{1}, x_{2}, \ldots\right], r 1_{1}, r 2_{1},\left[z_{1}\right]\right) \longrightarrow & r 1_{i+1}=f 1\left(x_{i}, r 1_{i}\right) ; \\
\left(\left[x_{2}, \ldots\right], r 1_{2}, r 2_{2},\left[z_{1}, z_{2}\right]\right) \longrightarrow & r 2_{i+1}=f 2\left(r 1_{i+1}, r 2_{i}\right) ; \\
\ldots & z_{i}=r 2_{i}
\end{array}
$$

Fig. 4. The behavior of the program in Figure 3. State is represented by quadruples where the first and final member are the contents of inQ and out $Q$, and the second and third members are the values of $r 1$ and $r 2$

The refined FSM in Figure 2 may be described by splitting our single rule into two rules: produce and consume, which communicate via the FIFO $q$ as shown in Figure 5.

The first thing to understand about this two-rule program is that it represents a nondeterministic specification which can be implemented by many different FSMs. For multiple rule programs, the semantics only state that any enabled rule (i.e., a rule in a state where its guard is true) can be executed; it does not determine which rule to choose if more than one is enabled. The following are possible schedules for this program:

$\begin{array}{ccc}\text { Schedule 1 } & \text { Schedule 2 } & \text { Schedule 3 } \\ \text { produce } & \text { produce } & \text { produce } \\ \text { consume } & \text { produce } & \text { produce } \\ \text { produce } & \text { consume } & \text { consume } \\ \text { consume } & \text { consume } & \text { produce } \\ \ldots & \ldots & \text { consume }\end{array}$

In the first schedule the program repeatedly enters a token into the FIFO and then immediately takes it out. This emulates the execution of the rule in the unrefined program (Figure 3) and leaves the FIFO $q$ empty after each consume rule execution. This schedule also does the same set of updates to registers $r 1$ and $r 2$ as the original program. The second schedule repeatedly queues up two tokens before removing them. Note that this schedule will be valid only if $q$ has space for two tokens. In the third schedule, except when the program
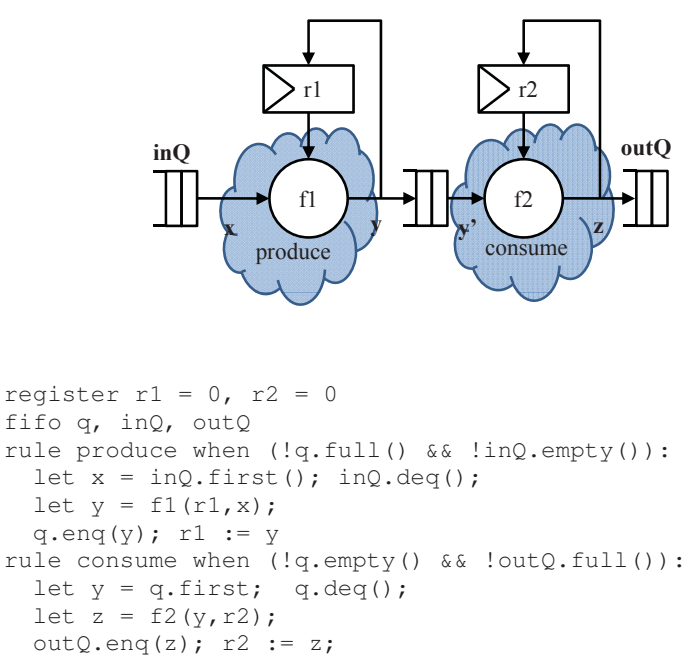

Fig. 5. A Refinement of the Program in Figure 3

starts, there will always be at least one token in $q$.

In case of multiple-rule programs, the behavior of the program must be thought of in terms of the set of permitted executions or more precisely, the set of the sequences of values assumed by various state elements. A scheduler picks a specific execution from this set. A schedule is chosen by the compiler (with voluntary inputs from the designer) based on some goodness criteria. The current Bluespec compiler [11] schedules as many enabled rules as possible every cycle as long as the rules do not conflict with each other. The behavior produced by a parallel scheduler must be consistent with some one-rule-at-time schedule. For the example at hand the Bluespec compiler will schedule only the producer in the first cycle and then repeatedly schedule consumer followed by producer in each subsequent cycle.

\section{Observability}

In what sense are the modules in Figure 3 and Figure 5 equivalent? Notice that given any sequence of inputs $x_{0}, x_{1}, x_{2}, x_{3}, \ldots$ both programs produce the same sequence of outputs $z_{1}, z_{2}, z_{3}, \ldots$. However, the interleavings of permissible "observations" are different. Assuming all FIFOs are of size 1, both systems can observe the following sequences: $x_{0}, z_{1}, x_{1}, z_{2}, x_{2}, z_{3} \ldots$ and $x_{0}, x_{1}, z_{1}, z_{2}, x_{2}, x_{3}, z_{3}, \ldots$. However, the sequence $x_{0}, x_{1}, x_{2}, z_{1}, z_{2}, z_{3}, \ldots$ can only be observed for the refined system, as the refined system has more buffering. Bluespec is expressive enough that one can write a program to distinguish between these two modules by only taking output from the module after a fixed number of inputs have entered. In spite of this, we want a notion of equivalence which permits this refinement.

The equality we will define applies only to full programs, i.e., those which do not interact with the outside world. To express equality between systems which interact with the outside world, we need to construct a "generic" context which represents all possible interactions with the outside world. This can be done naturally by adding sufficiently large source and 

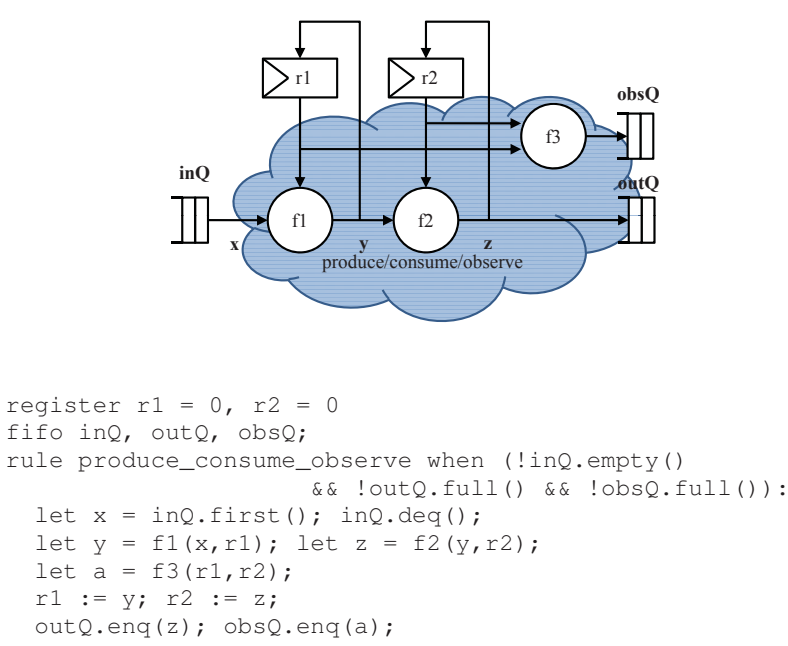

Fig. 6. Program of Figure 3 with an Observer

sink queues to drive interactions and store results. For instance, in our example we can attach a FIFO initially containing $N$ elements as source to inQ and a output FIFO with $M$ empty slots to outQ. It is easy to see why, given sufficient sizes of $N$ and $M$, this closed system will model all possible interactions of inQ and outQ with the outside world.

Under a weaker notion of equality, which relies on the transitive closure of rule applications instead of trace equivalence, the previously discussed refinement is correct. At the same time this weaker notion of equality can lead to errors if the module is used incorrectly (for instance if the input to the module changes depending on the number of values outstanding). We rely on the user to express desired distinctions programmatically. For instance if the user believes the relative order of inputs and outputs are necessary, he can add an additional FIFO to which we enqueue witnesses of both input and output events. We believe this is the right tradeoff between greater flexibility of refinements, and userresponsibility in expressing correctness [3].

As another example, consider refinements of a processor. To show the correctness of a refinement, it is sufficient to show that the refined processor generates the same sequence of instruction addresses of committed instructions as the original. As such we can add a single observation FIFO to the context to observe differences and consider all possible initial instruction and data memory configurations to verify correctness.

\section{An Example to Illustrate Incorrect Refinements}

While refinements are often easy to implement, it is not uncommon for a designer to make subtle mistakes. Consider the original one-rule produce-consume example augmented with observation logic as shown in Figure 6. In addition to doing the original computation, this program computes a function of the state of $r 1$ and $r 2$, and at each iteration inserts the result into a new FIFO queue(obsQ). A designer may want to do the same rule splitting exercise he had done with the first program, leading to the program in Figure 7.
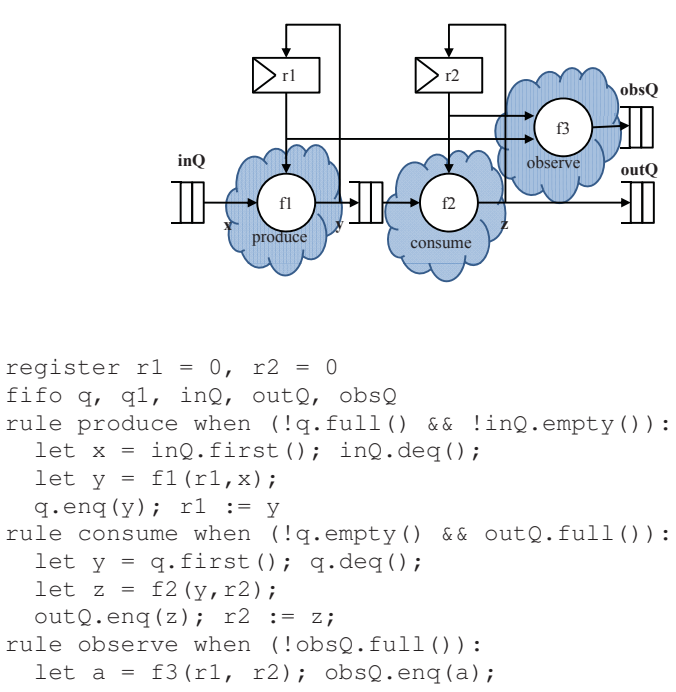

Fig. 7. An incorrect refinement of the program in Figure 6
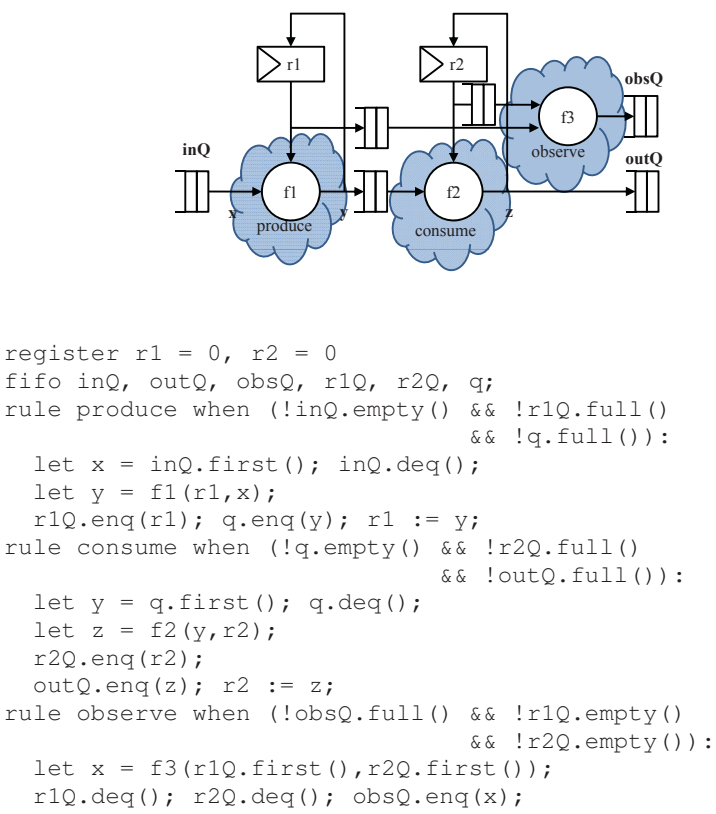

Fig. 8. A correct refinement of the program in Figure 6

This refinement is clearly wrong; we can observe $r 1$ and $r 2$ out-of-sync via the new observer circuit. Thus, the sequence produce observe consume has no correspondence in the original program. For our tool to be useful to a designer, it must be able to correctly determine that this refinement is incorrect (or rather that it failed to find a matching behavior in the original program). A correct refinement is shown in Figure 8 , where extra queues have been introduced to keep relevant values in sync. The correct solution would be obvious to an experienced hardware designer because all paths in a pipeline have the same number of stages.

\section{E. Refinements in Nondeterministic Programs}

The examples that we have considered so far have started with a single rule program. Such programs by definition 

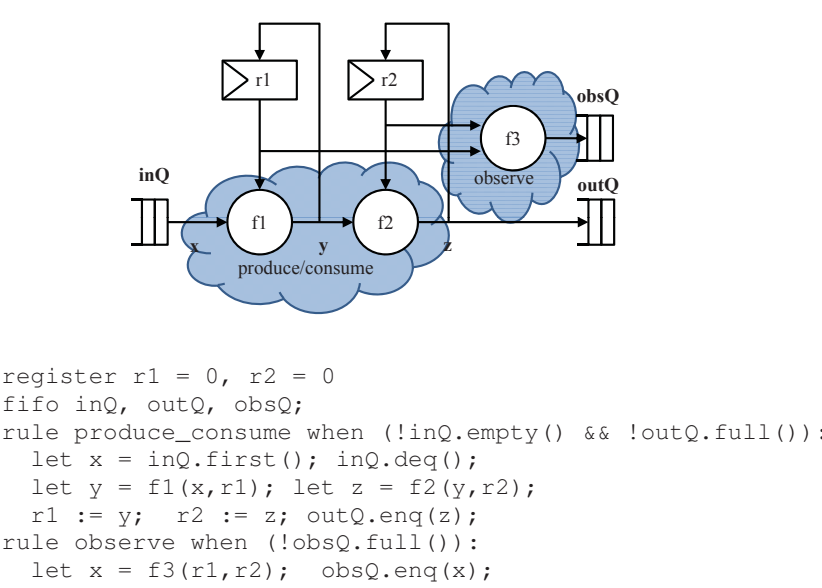

Fig. 9. A program with a nondeterministic observer

produce deterministic behaviors. Much of the value of rulebased programs comes from the ability to specify programs which can have multiple distinct behaviors. An example of a useful nondeterministic specification is that of a speculative processor whose correctness does not depend upon the number of instructions which are executed on the incorrect path. What does it mean to do a refinement in such a program?

Consider the example in Figure 9, which is a variation of our producer-consumer example with an observer (Figure 6). Unlike the lockstep version which did one observation for each iteration, in this program we are allowed to not only miss some updates of $r 1$ and $r 2$, but are permitted to repeatedly make the same observations. An implementation, i.e., a particular schedule, of this rule-based specification would pick some deterministic sequence of observations from the allowed set. By giving such a specification, the designer is saying, in effect, that any schedule of observations is acceptable. In that sense, the observations made in the program in Figure 6 are an acceptable implementation of this nondeterministic program. By the same reasoning we could argue that the refinement shown in Figure 8 is a correct refinement of Figure 9.

But suppose we did not want to rule out any behaviors prematurely in our refinements, then a correct refinement will have to preserve all possible behaviors. We show a correct refinement of the nondeterministic program in Figure 10, where we introduce an extra register, $r 1 \mathrm{p}$, to keep a relevant copy of $r 1$ in sync with $r 2$ with which to make legal observations.

It is nontrivial to show that all behaviors in the new program can be modeled by the original nondeterministic specification and vice versa. As we demonstrate later, our tool can automatically verify this condition, though we do require the programmer to specify a projection function, by which state in the two different programs can be related. The partial function relationship is both natural for designers to come up with and easy to specify. Having manually defined this function, the designer passes it to the tool which tells him either that the refinement is correct, or if it is not, returns
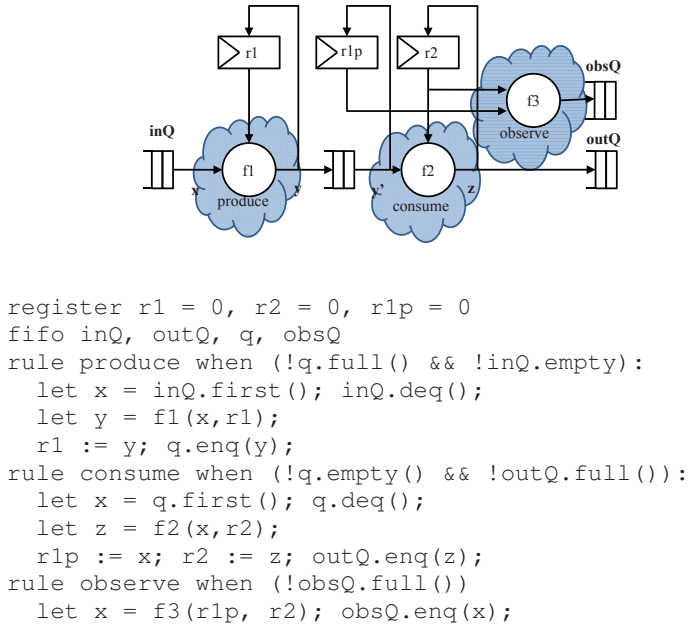

Fig. 10. Correct refinement of Figure 9

an execution from one program which it believes cannot be simulated by the other.

\section{Formalizing BehaViors AND CORRECTNESS OF REFINEMENTS}

We model the behavior of a program using a state transition system. A program $P$ has a collection of state elements and a set of rules $R_{P}$. The states in the transition system are the values assumed by these state elements. Thus, the state transition system of a program with two 32-bit registers would have $2^{64}$ states.

\section{A. Equivalence of Programs}

Definition 1 (State Transition System of Program $P$ ). Each program $P$ is modeled by a state transition system given by a triple of the form:

$$
\left(S, S_{0}, \longrightarrow\right)
$$

where $S$ is the set of states associated with program $P ; S_{0} \subseteq S$ is the set of states corresponding to initial configurations of $P$; and $\longrightarrow \mathcal{S} \subseteq S \times S$ is the transition relation, defined such that $\left(s, s^{\prime}\right) \in \longrightarrow \mathcal{S}$ if and only if there exists some rule $R$ in $P$ whose execution takes the state $s$ to $s^{\prime}$. In addition, we write $\rightarrow$ to denote the reflexive transitive closure of $\longrightarrow$.

It is sometimes useful to know which rule $R$ caused the transition from $s$ to $s^{\prime}$; we will denote this by writing:

$$
s \stackrel{R}{\longrightarrow} s^{\prime}
$$

Similarly we write the sequence of rule executions $\sigma=$ $R_{1}, R_{2}, \ldots R_{n}$ where:

$$
s_{0} \stackrel{R_{1}}{\longrightarrow} s_{1} \stackrel{R_{2}}{\longrightarrow} s_{2} \ldots \stackrel{R_{n}}{\longrightarrow} s_{n}
$$

as: 


$$
s_{0} \stackrel{\sigma}{\rightarrow} s_{n}
$$

Intuitively two programs $P_{1}$ and $P_{2}$ with the same set of states are equivalent if every transition in one system can be simulated by a sequence of transitions in the other. That is, every finite execution $s \rightarrow s^{\prime}$ in $P_{1}$ has a corresponding execution $s \rightarrow s^{\prime}$ in $P_{2}$ and vice versa.

Definition 2 (Equivalence of Programs). Let $P$ be a program modeled by the transition system $\mathcal{S}=\left(S, S_{0}, \longrightarrow \mathcal{S}\right)$ and let $P^{\prime}$ be a program modeled by the transition system $\mathcal{S}^{\prime}=\left(S^{\prime}, S_{0}^{\prime}, \longrightarrow \mathcal{S}^{\prime}\right) ; P$ and $P^{\prime}$ are are equivalent if and only if $S=S^{\prime}, S_{0}=S_{0}^{\prime}$, and $\rightarrow_{S}=\rightarrow_{S^{\prime}}$.

This definition captures the fact that two program may have a different set of rules but may still be equivalent in terms of their transitive closure. This ability allows us to add "derived" rules whose execution is always expressible in terms of the other rules in the program without affecting the meaning of the program, which is very important in implementing these systems. Sometimes the consequences of this equality are nonintuitive. For example, a modulo up-counter ( 0 to 1 to 2 back to 0 ) and a modulo down-counter ( 0 to 2 to 1 back to 0 ) will have the same transitive closure and thus be considered equivalent. Our notion of equality only says that you can get from 0 to 1 , and whether you did so directly or through another state, i.e., 2 does not matter. If we want to distinguish these two counters, we can always add an observation queue to record the counter value after each transition. Practically, this is not required in a real system as the context in which such a counter is used will implicitly either demand one of these orders or will work equally well with either counter.

Modeling the behavior of a program in terms of the transitive closure of executions also allows us to define several other notions precisely:

Definition 3 (Deterministic Programs). Let $P$ be a program modeled by the transition system $\mathcal{S}=\left(S, S_{0}, \longrightarrow_{\mathcal{S}}\right) . P$ is deterministic if and only if all pair-wise executions are joinable, that is: when for all $s_{0}, s_{1}, s_{2} \in S$ such that $s_{0} \rightarrow \mathcal{S} s_{1}$ and $s_{0} \rightarrow \mathcal{S} s_{2}$, there exists an $s_{3} \in S$ such that $s_{1} \rightarrow \mathcal{S} s_{3}$ and $s_{2} \rightarrow \mathcal{S} s_{3}$. A program is called nondeterministic if it is not a deterministic program.

Examples of nondeterministic programs are shown in Figures 7, 9, and 10. All other examples given in Section II are deterministic according to the above definition.

As we have shown, implementing a rule-based program requires choosing a schedule. A scheduler by definition implements a specific execution sequence. Thus, in the case of non-deterministic programs the scheduler eliminates all nondeterminism and produces one specific behavior from among the allowed set of behaviors. Even for deterministic programs the scheduler is sometimes not able to produce a "complete" behavior because the scheduling of rules may be unfair. In such cases we say that an implementation is partially correct:
Definition 4 (Partially Correct Implementation). Let $P$ be a program modeled by the transition system $\mathcal{S}=\left(S, S_{0}, \longrightarrow_{\mathcal{S}}\right)$ and let $P^{\prime}$ be a program modeled by the transition system $\mathcal{S}^{\prime}=\left(S^{\prime}, S_{0}^{\prime}, \longrightarrow \mathcal{S}^{\prime}\right) . P^{\prime}$ is a partially correct implementation of $P$ if and only if $S^{\prime}=S, S_{0}^{\prime}=S_{0}$, and $\rightarrow \mathcal{S}^{\prime} \subseteq \rightarrow \mathcal{S}$.

\section{B. Correctness of Refinements}

Correctness is slightly more complicated to define for the refinements because the specification and implementation programs have different state elements. In addition, the set of rules in the implementation is formed by removing a rule from the specification rule set, replacing it by several rules which together simulate the removed rule. The new rules operate on the new implementation state, and the remaining rules are "lifted" to operate on the new implementation state. This is clearly more complicated than the simple addition of a derived rule described in the previous section.

Consider the refinement from the program in Figure 3 to the one Figure 5. Both programs have $r 1$ and $r 2$ but they get updated at different times in the two programs and may not match. Intuitively we know that if $q$ contains an element, then we are part way through at least one round of an equivalent produce_consume atomic computation, and $r 1$ and $r 2$ will appear out of sync in the two programs. Conversely, whenever $q$ is empty we should be able to draw a correspondence between the executions of the two programs.

We can guarantee that the refined program does not add new behaviors if we can show that for any execution in the refined program, whenever it reaches a state where $q$ is empty we can find a corresponding execution in the original program which has the same values for the matching state elements, i.e., $r 1$ and $r 2$. To guarantee that we haven't lost behaviors we must also show the converse: namely that any computation in the original program can be mimicked by the refined program. This is quite easy to show because produce followed by consume behaves exactly as produce_consume.

We first offer an intuitive reason why the original program can mimic the refined one. Consider those prefixes of a schedule in the refined program which have equal number of produce and consume rule executions. At the end of such a prefix, $q$ must be empty and we can meaningfully verify that the specification and implementation match. Further, since produce always adds a token and consume always removes one, we must have a non-empty $q$ when we have an unequal number of produces and consumes. As such we there's no meaningful state match. However, by making an appropriate number of consumes we can always empty $q$ where upon our previous line of reasoning would apply and we'd have a match. Therefore, all we have to show is that 1) for all prefixes where we end with an empty $q$ the specification can mimic the execution of the implementation, and 2) for all other sequences the implementation can move to a state where $q$ is empty.

To reason about the correctness of refinement formally, we need a projection function $p$ to relate implementation state to specification state. This projection function is often 
partial. The states which are in the domain of the projection function (e.g., $\operatorname{Dom}(p))$ are called relatable. That is, if $\mathcal{S}=\left(S, S_{0}, \longrightarrow \mathcal{S}\right)$ is refined by $\mathcal{T}=\left(T, T_{0}, \longrightarrow \mathcal{T}\right)$ by a partial-function $p: T \rightarrow S$, the set of relatable states in $\mathcal{T}$ is, by definition, the set $\operatorname{Dom}(p)$ of states where $p$ is defined. All initial states should be in $\operatorname{Dom}(p)$, that is, $T_{0} \subseteq \operatorname{Dom}(p)$. Furthermore, for any two states $t_{1}, t_{2} \in \operatorname{Dom}(p)$ such that $t_{1} \rightarrow \mathcal{T} t_{2}$, we should have $p\left(t_{1}\right) \rightarrow_{\mathcal{S}} p\left(t_{2}\right)$.

This condition covers all finite executions in the implementation program which start and end in relatable states, but not those which start in a relatable state but do not end in one. To address those executions, we must verify that every finite execution in the implementation beginning in a relatable state which does not end in a relatable state, can eventually reach one.

Definition 5 (Partially Correct Refinement). Let $P$ be a program modeled by the transition system $\mathcal{S}=\left(S, S_{0}, \longrightarrow \mathcal{S}\right)$, $P^{\prime}$ be a program modeled by the transition system $\mathcal{T}=$ $\left(T, T_{0}, \longrightarrow \mathcal{T}\right)$, and $p: T \rightarrow S$ a partial function relating states having the property that $T_{0} \subseteq \operatorname{Dom}(p) . P^{\prime}$ is a partially correct refinement of $P$ exactly when the following conditions hold:

1) Correspondence of Initial State: $\left\{p(t) \mid t \in T_{0}\right\}=S_{0}$.

2) Soundness: For all $t_{1}, t_{2} \in \operatorname{Dom}(p)$ such that $t_{1} \rightarrow \mathcal{T} t_{2}$, also $p\left(t_{1}\right) \rightarrow \mathcal{s} p\left(t_{2}\right)$.

3) Limited Divergence: For all $t_{0} \in T_{0}$ and $t_{1} \in T$ such that $t_{0} \rightarrow \mathcal{T} t_{1}$, there exists $t_{2} \in \operatorname{Dom}(p)$ such that $t_{1} \rightarrow \mathcal{T} t_{2}$.

The first clause states the initial states correspond to each other. The second clause states that every possible execution in the implementation whose starting and ending states have corresponding states in the specification must have a corresponding execution in the specification. The third clause states that from any reachable state in the implementation we can always get back to a state which corresponds to a state in the specification. Note that these clauses alone do not guarantee that the specification has been fully implemented. To guarantee that a specification has been fully implemented, we need the notion of total correctness.

Definition 6 (Totally Correct Refinement). A totally correct refinement is a partially correct refinement that, in addition, satisfies:

4) Completeness: For all $s_{1}, s_{2} \in S$ and $t_{1} \in \operatorname{Dom}(p)$ such that $s_{1} \rightarrow \mathcal{S} s_{2}$ and $p\left(t_{1}\right)=s_{1}$, there exists an $t_{2} \in \operatorname{Dom}(p)$ such that $t_{1} \rightarrow \mathcal{T} t_{2}$ with $p\left(t_{2}\right)=s_{2}$.

This states that all executions in the specification program are preserved in the implementation.

Of the conditions for total correctness, correspondence of initial state the completeness are easy to verify in the context of rule splitting. This leaves us only concerned with soundness and limited divergence.

\section{Checking Simulation Using SMT Solvers}

We can understand the execution of rule $R$ as the application of a pure function $f_{R}$ of type $S \longrightarrow S$ to the current state. When the guard of $R$ fails, it causes no state change (i.e., $f_{R}(s)=s$ ). We can compose these functions to generate a function $f_{\sigma}$ corresponding to a sequence of rules $\sigma$. To prove the correctness of refinements, we pose queries about $f_{\sigma}$ to an SMT solver.

SMT solvers are conceptually Boolean Satisfiability (SAT) solvers extended to allow predicates relating to non-boolean domains (characterized by the particular theories it implements). SMT solvers do not directly reason about computation, but rather permit assertions about the input and output relation of functions. They provide concrete counter-examples when the assertion is false. For example, suppose we wish to verify that some concrete function $f$ behaves as the identity function. We can formulate a universal quantification representing the property: $\forall x, y \cdot(x=f(y)) \wedge(x=y)$. An SMT solver can be used to solve this query, provided the domains of $x$ and $y$ are finite, and $f$ is expressed in terms of boolean variables. If the SMT solver can find a counter-example, then the property is false. If not, then we are assured that $f$ must be the identity. The speed of SMT solvers on large domains is due to their ability to exploit symmetries in the search space [6].

When we reason about rule execution it is often useful to discard all executions where a rule produces no state update (a degenerate execution); it is clearly equivalent to the same execution with that rule removed. As such, when posing questions to the solver it is useful to add clauses which state that sequential states of an execution are different. To represent this assertion for the rule $R$, we define the predicate function $\hat{f}_{R}\left(s_{2}, s_{1}\right)$ which asserts that the guard of rule $R$ evaluates to true in $s_{1}$ and that $s_{2}$ is the updated state:

$$
\hat{f}_{R}\left(s_{2}, s_{1}\right)=\left(s_{2}=f_{R}\left(s_{1}\right)\right) \wedge\left(s_{2} \neq s_{1}\right)
$$

As with the functions, we can construct a larger predicate $\hat{f}_{\sigma}\left(s_{2}, s_{1}\right)$ which is true when a non-degenerate execution of $\sigma$ takes us from $s_{1}$ to $s_{2}$.

Now we explain how the propositions in Definition 5 can be checked via a small set of easily answerable SMT queries.

\section{A. Checking Correctness}

For this discussion let us assume we have a specification program $P$ and a refinement $P^{\prime}$ and their respective transition systems $\mathcal{S}=\left(S, S_{0}, \longrightarrow_{\mathcal{S}}, \rightarrow_{\mathcal{S}}\right)$ and $\mathcal{T}=\left(T, T_{0}, \longrightarrow \mathcal{T}, \rightarrow_{\mathcal{T}}\right)$ are related by the projection function $p: T \rightarrow S$.

Now let us consider the soundness proposition from Definition 5: $\forall t_{1}, t_{2} \in \operatorname{Dom}(p) .\left(t_{1} \rightarrow \mathcal{T} t_{2}\right) \Longrightarrow\left(p\left(t_{1}\right) \rightarrow_{\mathcal{S}} p\left(t_{2}\right)\right)$.

A naïve approach to verifying this property entails explicitly enumerating all pairs $\left(t_{1}, t_{2}\right)$ in the relation $\rightarrow \mathcal{T}$ and checking the corresponding pair $\left(p\left(t_{1}\right), p\left(t_{2}\right)\right)$ in the relation $\rightarrow \mathcal{s}$. As the set of states in both systems are finite, both of these relations are similarly finite (bounded by $|T|^{2}$ and $|S|^{2}$, respectively) and thus we can mechanically check the implication.

We can substantially reduce this work by noticing two facts. First, because of transitivity, if we have already checked the correctness of $t_{1} \stackrel{\sigma_{1}}{\rightarrow} \mathcal{T} t_{2}$ and $t_{2} \stackrel{\sigma_{2}}{\rightarrow} \mathcal{T} t_{3}$, then there is no need 
to check the correctness of execution $\sigma=\sigma_{1} \sigma_{2}$. Second, if we have already found an execution $\sigma$ such that $t \stackrel{\sigma}{\rightarrow} \mathcal{T} t^{\prime}$ then we can ignore all other executions $\sigma^{\prime} \neq \sigma$ which have the same starting and ending states as they must also be correct. This essentially reduces the task from checking the entire transitive closure to checking only a covering of it. Unfortunately, the size of this covering is still very large.

The insight on which our algorithm is built is that proving this property for a small set of finite rule sequences is tantamount to proving the property for any execution. We explain this idea using the program in Figure 5.

- Let's begin by considering all rule sequences of length one: produce and consume.

- The sequence consume is never valid for execution starting in a relatable state so we need not consider it further.

- The sequence produce is valid to execute but does not take us to a relatable state, so we construct more sequences by extending it with each rule in the implementation. These new sequences are produce produce and produce consume.

- The sequence produce consume always takes a relatable state to another relatable state. We check that all concrete executions of produce consume have a corresponding execution in the specification. We do this check over a finite set of sequences in $\mathcal{S}$ (in this case: produce_consume), the selection of which we will explain later. Since all executions of produce consume end in a relatable state, we need not extend it.

- produce produce never takes us from relatable state to relatable state, so again extend the sequence to get new sequences produce produce produce and produce produce consume.

- produce produce produce is degenerate if $q$ is of length 2 ( $q$ has to have some known finite length).

- Suppose we could prove that the sequence produce produce consume always behaves like produce consume produce. Then any execution prefixed by produce produce consume is equal to an execution prefixed by produce consume produce. Notice that we need not consider any sequences prefixed by produce consume produce because itself has the prefix produce consume. Therefore we need not consider further sequences prefixed by produce produce consume.

- Because we have no new extension to consider, we have proved the correctness of this refinement.

Each of these steps involved an invocation of the SMT solver on queries which are much simpler than the general query presented previously, though the solver still must conceptually traverse the entire state space. The queries themselves are simple because they are always presented using rule sequences of concrete length, which are much smaller than the sequences in $\rightarrow \mathcal{T}$. The only problem with this procedure is that in the worst case this algorithm will run for the maximum number of states in $\mathcal{S}$. If we give up before the correctly terminating condition, this only means we have failed to establish the correctness of the refinement. We think it is unlikely that the type of refinements we consider in this paper will enter this case. In fact most refinements can be shown to be correct with very small number of considered sequences.

\section{B. The Algorithm}

The algorithm constructs three sets, each of whose elements corresponds to a set of finite executions of $\mathcal{T}$. For each iteration, $R_{\sigma}$ represents the set of finite sequences for which we have explicitly found a corresponding member, and $U$ represents the set of finite executions we have yet to verify (each element of $U$ conceptually represents all finite sequences starting with some concrete sequence of rule executions $\sigma$ ). $N U$ is the new value of $U$ being constructed for the next iteration of the execution.

\section{The Verification Algorithm:}

1) Initially: $R_{\sigma}:=\emptyset, U:=\left\{R_{i} \mid R_{i} \in R_{P^{\prime}}\right\}, N U:=\emptyset$

2) if $U=\emptyset$, we have verified all finite executions. Exit with Success.

3) Check if we have reached our iteration limit. If so, give up, citing the current $U$ set as the cause of the uncertainty.

4) For each $\sigma \in U$ :

a) Check if the execution of $\sigma$ from a relatable state is ever non-degenerate:

$\exists t_{1} \in T, t_{2} \in \operatorname{Dom}(p) .\left(t_{1} \stackrel{\sigma}{\rightarrow} \mathcal{T} t_{2}\right)$

If no execution exists we can stop considering $\sigma$ immediately.

b) Check if $\sigma$ should be added to $R_{\sigma}$. That is, if some execution of $\sigma$ should have a correspondence in $\mathcal{S}$ :

$\exists t, t^{\prime} \in \operatorname{Dom}(p) .\left(t \stackrel{\sigma}{\rightarrow} \mathcal{T} t^{\prime}\right)$

If so $R_{\sigma}:=R_{\sigma} \cup\{\sigma\}$.

c) Check if all finite executions of $\sigma$ that should have a correspondence in $\mathcal{S}$ have such a correspondence:

$\forall t, t^{\prime} \in \operatorname{Dom}(p) .\left(t \stackrel{\sigma}{\rightarrow} \mathcal{T} t^{\prime}\right) \Longrightarrow \exists \sigma^{\prime} \cdot\left(p(t) \stackrel{\sigma^{\prime}}{\rightarrow} \mathcal{S} p\left(t^{\prime}\right)\right)$ If this fails due to some concrete execution of $\sigma$, exit with Failure providing the counter example as justification.

d) For every execution where $\sigma$ does not put us in a relatable state, we must show that extensions of the form $\sigma \sigma^{\prime}$ have an equivalent execution $\sigma_{1} \sigma_{2} \sigma^{\prime}$, where $\sigma_{1}$ is a member of $R_{\sigma}$ and $\left|\sigma_{1} \sigma_{2}\right| \leq|\sigma|$. Thus, the correctness of $\sigma \sigma^{\prime}$ is reduced to the correctness of the shorter sequence $\sigma_{2} \sigma^{\prime}$.

$$
\begin{gathered}
\forall t \in \operatorname{Dom}(p), t^{\prime} \in T .(t \stackrel{\sigma}{\rightarrow} \mathcal{T}) \Longrightarrow \\
\exists \sigma_{1} \in R_{\sigma}, \sigma_{2} . \\
\left(\left|\sigma_{1} \sigma_{2}\right| \leq|\sigma|\right) \wedge\left(\sigma_{1}(t) \in \operatorname{Dom}(p)\right) \\
\wedge\left(\sigma_{2}\left(\sigma_{1}(t)\right)=t^{\prime}\right) .
\end{gathered}
$$

If this succeeds, we need not consider executions for which $\sigma$ is a prefix. If not, partition all the extensions into the $\left|R_{P^{\prime}}\right|$ sets of rules by extending $\sigma$ by one rule execution. $N U:=N U \cup\left\{\sigma \cdot R_{i} \mid R_{i} \in R_{\mathcal{P}^{\prime}}\right\}$.

5) $U:=N U, N U:=\emptyset$, Go to Step 2. 


\section{Formulating the SMT Queries}

The four conditions in the inner-most loop of the algorithm can be formulated as the following SMT queries using the $\hat{f}_{\sigma}$ predicate and the computational version of projection function $p, \hat{p}: T \longrightarrow S$ and $\mathrm{rel}: T \longrightarrow\{0,1\}$ where $p$ and $\hat{p}$ are the same if $p$ is defined and $\operatorname{rel}(t)$ returns true exactly when $p(t)$ is defined.

1) Existence of valid execution of $\sigma$ starting from a relatable state:

$\exists t_{1}, t_{2} \in T \cdot \hat{f}_{\sigma}\left(t_{2}, t_{1}\right) \wedge \operatorname{rel}\left(t_{1}\right)$

2) Verifying that each execution of $\sigma$ in the implementation starting and ending in a relatable state has a corresponding execution in the specification:

$\forall t_{1}, t_{2} \in T$.

$$
\begin{gathered}
\left(\operatorname{rel}\left(t_{1}\right) \wedge \operatorname{rel}\left(t_{2}\right) \wedge \hat{f}_{\sigma}\left(t_{2}, t_{1}\right)\right) \Longrightarrow \\
\bigvee_{\sigma^{\prime} \in E C(\sigma)}\left(\hat{f}_{\sigma^{\prime}}\left(\hat{p}\left(t_{2}\right), \hat{p}\left(t_{1}\right)\right)\right)
\end{gathered}
$$

where $E C$ is the "expected correspondences" function which takes a sequences of rules $\sigma$ in $\mathcal{T}$ and returns a finite set of sequences in $\mathcal{S}$ to which $\sigma$ is likely to correspond. This function can be easily generated by the tool or the user, since the refinements are rule splitting, it is easy to predict the candidates in the specification that could possibly mimic $\sigma$. For instance, consider the refinement of the program in Figure 3 to the one in Figure 5. Each occurrence of produce in the implementation should correspond to an occurrence of produce_consume in the specification. Thus, the sequence produce produce consume produce, if it has a correspondence at all, could only correspond to the sequence produce_consume produce_consume produce_consume.

3) Checking that every valid execution of $\sigma$ in the implementation has an equivalent sequence which is correct by concatenation of smaller sequences:

$\forall t_{1}, t_{2}, t_{m} \in T$. $\operatorname{rel}\left(t_{1}\right) \wedge \hat{f}_{\sigma}\left(t_{2}, t_{1}\right) \Longrightarrow \operatorname{rel}\left(t_{m}\right) \wedge$ $\bigvee_{\sigma_{1} \in R_{\sigma}}\left(\bigvee_{\sigma_{2} \in E A\left(\sigma, \sigma_{1}\right)}\left(\hat{f}_{\sigma_{1}}\left(t_{m}, t_{1}\right) \wedge \hat{f}_{\sigma_{2}}\left(t_{2}, t_{m}\right)\right)\right)$

Our algorithm requires us to find, given $\sigma$ and $\sigma_{1}$ in $\mathcal{T}$, a $\sigma_{2}$ such that the execution of $\sigma$ is the same as the execution of $\sigma_{1} \sigma_{2}$, and $\left|\sigma_{1} \sigma_{2}\right| \leq|\sigma|$. We will assume the existence of a "expected alternatives" function $E A$ which enumerates all possible $\sigma_{2}$ given $\sigma$ and $\sigma_{1}$.

\section{Step-By-Step Demonstration}

For the sake of clarity, we provide an additional example of the algorithm's execution. Figure 11 gives the trace of reasoning through which our algorithm progresses in order to verify the refinement of the program in Figure 6 to the one in Figure 7. Each node represents an element in the algorithm's set $U$, and the path from the root to any node in the graph corresponds to the concrete value $\sigma$ for that node. At each node, we verify the correctness of all corresponding finite executions of $\sigma$ : nodes displayed as $\perp$ are vacuously true by Step $4 \mathrm{a}$, while other leaf nodes are either true by Step $4 \mathrm{~d}$ or incorrect by Step 4c. The program is ultimately rejected as the refinement being checked is incorrect:

- We begin by considering all rule sequences of length one executed in a relatable state: produce, consume, and observe. The rule observe always ends in a relatable state, and corresponds directly to the observe rule in the specification program. consume is never valid to execute, so the only sequence which we extend is produce since it never ends in a relatable state.

- We now extend produce, giving us three new sequences to consider: produce produce, produce consume, and produce observe. produce consume always ends in a relatable state and corresponds to the execution of produce_consume in the specification. Neither produce produce, nor produce observe ever end in a relatable state, and since we are unable to prove their equivalence to an execution we have already verified, we extend both.

- In the third iteration, we consider the sequence produce observe consume, which always ends in a relatable state. This exposes an error in the refinement since there is no possible sequence of rule in the specification which produces this final state (in this case, the implementation enqueues a value to obsQ which the specification is unable to replicate.

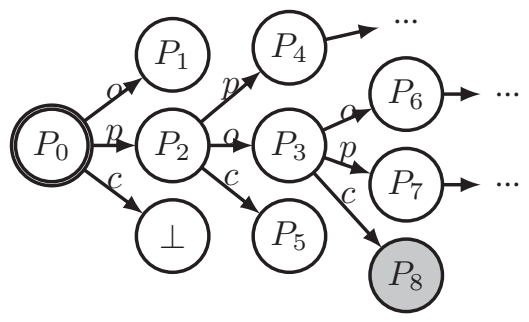

Fig. 11. Tree visualization of the algorithmic steps to check the refinement of the program in Figure 6 to the one in Figure 7

\section{The Debugging Tool and Evaluation}

Our tool works with Bluespec SystemVerilog (BSV) [4] which is a commercial rule-based language aimed at hardware design. It takes as input an intermediate output of the BSV compiler where all data types are represented as bit vectors. The rules are expressed using bit vector expressions and primitive modules (e.g., registers, FIFOs, and memories). The algorithm in Section IV works more efficiently when rule sizes are small, therefore the first phase of the tool is to reduce the size of actions by action sequentialization, conditional merging, and "when lifting" [7]. Next, the tool generates the function $f_{R}$ for each rule $R$. We use typed $\lambda$-calculus with let blocks to represent these functions and apply many small transformations to simplify them.

The tool is essentially an embodiment of the algorithm shown in Section IV in Haskell. As we have discussed, this algorithm makes many queries to an SMT solver; we use the STP SMT solver [10] for this purpose. By static analysis 


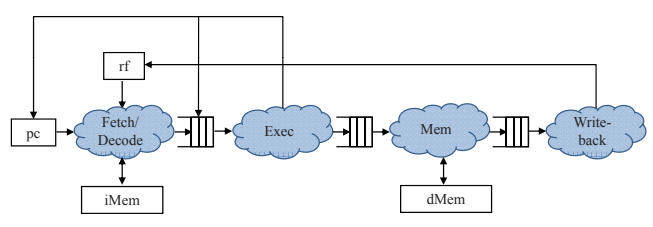

(a) 4 stage SMIPS Processor

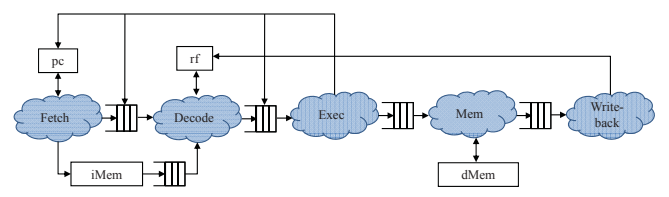

(b) 5 stage SMIPS Processor

Fig. 12. SMIPS processor refinement

(e.g., rule commutativity and sequence degeneracy) of the programs, we remove unneeded sequences from consideration in the sets $E A$ and $E C$. This has substantial impact on the size of SMT queries.

To demonstrate our tool, we consider a refinement of a Simplified MIPS (SMIPS) processor, whose ISA contains a representative subset of 35 instructions from the MIPS ISA. While the ISA semantics are specified one instruction at a time, our program is pipelined with five stages in the style of the DLX processor [17], and resembles soft-cores used in many FPGA designs. The execution of the final implementation is split into the following five separate stages (see Figure 12(b)):

1) Fetch requests the next instruction from the instruction memory (imem) based on the pc register which it then updates speculatively to the next consecutive pc.

2) Decode takes the data from the instruction memory and the fetch stage, decodes the instruction, and passes it along to the execute stage. It also reads the appropriate locations in the register file $r f$, stalling to avoid data hazards (stall logic is not shown).

3) Execute gets decoded instructions from the execute queue, performs ALU operations and translates addresses for memory operations. To handle branch operations, it kills mispredicted instructions and sets the pc.

4) Memory performs reads and writes to the data memory, passing the data to the writeback state. (A further refinement might introduce a more realistic split-phase memory, which would move some of this functionality into the writeback stage).

5) Writeback gets instructions in the form of register destination and value pairs, performing the update on the register file.

The implementation program contains one rule per stage, and stages communicate via FIFO connections. If we were to executes the rules for each stage in reverse order (starting from writeback and finishing with fetch), the result is a fully pipelined system. If each FIFO is implemented as a single register with a valid bit, this is indistinguishable from the standard processor complete with pipeline stalls. If instead we execute the rules in pipeline order, we end up with a system where the instructions fly through the processor one-at-a-time. For code simplicity, our final implementation actually decomposes the execute stage into three mutually exclusive cases, implementing each with a separate rule(exec, exec_branch, and exec_branch_mispredict). Since the rule guards are mutually exclusive, this does not modify the pipeline structure, nor does it change the analysis.

Our implementation is relatively complicated and we would like to know if it matches the ISA. One way to achieve this is to start with a single-rule description of the behavior (transliterated directly from the documentation, which we consider to be correct), and incrementally refine the program towards the final five-stage implementation. After each refinement, our tool can be used to verify correctness with regards to the previous iteration. For the sake of brevity, we examine only the final refinement, which takes a four-stage processor (Figure 12(a)) and splits the fetch-decode stage. Though the transformation is straightforward, the tool must be able to correctly resolve the effect of speculative execution from branch prediction.

The tool is able to establish the correctness of this refinement step in under 7 minutes. To do so it needed to check 21 executions in the refined program of maximum length 3, finding correspondences in the four-stage program for the 5 corresponding rules, fetch_decode for fetch decode, and exec_branch_mispredict for the mispeculating sequences fetch exec_branch_mispredict and fetch fetch exec_branch_mispredict.

\section{RELATED WORK}

There is a rich body of literature for verifying pipelined processors (see,e.g., [2], [5], [12], [15], [20]). Most of this work is motivated by proving the correctness of a pipelined or our-of-order microarchitectural implementation of a processor. Usually the specification is an unpipelined model of the processor. The work generally relies on mechanical theorem proving, and the technique of pipeline draining to match the specification and implementation states is well established in this context. Our lifting and projection functions effectively do the same thing, and at some level, all these papers are about the ability of the systems to simulate each other. The literature on bisimulation (see, e.g., [16]) and stuttering simulation (see, e.g., [14]) is also very rich and relevant.

Our tool is totally automatic and is intended as a debugging aid as opposed to establishing total correctness of the design. The problem we address is one of local transformation, i.e., an atomic rule which has been split into multiple atomic subrules with the same functionality. We want to show that this local transformation has no "bad" consequences on the whole design. In this scenario almost every aspect of the design has been specified and it becomes amenable to automated verification. The proof of the refinements we discussed relied on a concrete size for the inserted FIFOs; in contrast, the proof of Arvind and Shen [2] works for FIFOs of any size. 
Singh et. al. [19] translate a restricted subset of Bluespec to PROMELA as a means for querying the SPIN model checker about refinements. Conceptually one could translate both the specification and implementation programs into PROMELA and verify that the PROMELA systems represent a valid PROMELA refinement. The subset of Bluespec they consider is interesting from a semantic point of view, but not large enough to deal with realistic programs. Richards et. al. [18] proposed a more complete translation of Bluespec to PVS leveraging a monadic representation. Both of these focused on the task of getting the Bluespec design translated for the model checker faithfully. Expressing our formulation as correctness of refinements via the transitive closure of rules as an LTL or CTL property is not straightforward; in fact it is not clear to us if this is even possible.

\section{DISCUSSION}

In this paper we have presented both a notion of refinement and a tool for verifying the correctness of such refinements. The exact notion of correctness is aimed at being easily and naturally expressible by a designer. The tool quickly finds the correspondence by searching for a minimum cover of the paths in the system starting from all possible relatable states.

The notion of equality we implement is that of a closed system. We have shown intuitively how to rephrase the verification of open system, i.e., modules, by encoding the notion of equivalence programmatically. This is highly practical as it is both easy to do and lends itself to the way that designers understand equivalences, allowing them to express their concerns more precisely. It is possible to automatically generate the necessary contexts to express some standard equivalences, e.g., trace equivalence.

The current tool can be improved in three orthogonal dimensions. First, we currently only leverage the theory of bit vectors. By adding additional theories of FIFOs, arrays, and uninterpreted functions [13] we can dramatically reduce the complexity of our SMT queries. Secondly, our interface with the SMT solver is inefficient, requiring file-level IO. More than half of the compute time comes from marshaling and unmarshaling the query representation. This clearly can be eliminated by directly integrating an SMT solver with our tool. Finally, our algorithm allows us to reason about each element of $U$ in parallel. This is quite straightforward to exploit in a multithreaded implementation of our program. With a fast enough tool, we are confident that this can help shape how designers approach their work and will encourage further use of formal reasoning in design.

\section{ACKNOWLEDGMENTS}

We are thankful to the anonymous referee who helped us clarify the difference between our transitive closure-based equivalence and the more standard trace-based techniques. We are thankful to Armando Solar-Lezama for helping us understand the difficulty of expressing our problem in model checking. This work has been supported by the National Science Foundation (\#CCF-0541164).

\section{REFERENCES}

[1] Arvind, Rishiyur S. Nikhil, Daniel L. Rosenband, and Nirav Dave. Highlevel Synthesis: An Essential Ingredient for Designing Complex ASICs. In Proceedings of ICCAD'04, San Jose, CA, 2004.

[2] Arvind and Xiaowei Shen. Using Term Rewriting Systems to Design and Verify Processors. IEEE Micro, 19(3):36-46, May 1999.

[3] Arvind and Nirav Dave and Michael Katelman. Getting formal verification into design flow. In Proceedings of the 15th international symposium on Formal Methods, FM '08, pages 12-32, 2008.

[4] Bluespec, Inc., Waltham, MA. Bluespec SystemVerilog Version 3.8 Reference Guide, November 2004

[5] Jerry R. Burch and David L. Dill. Automatic verification of pipelined microprocessor control. In Computer Aided Verification, pages 68-80, 1994.

[6] Stephen A. Cook. The complexity of theorem-proving procedures. In Proceedings of the third annual ACM symposium on Theory of computing, STOC '71, pages 151-158, New York, NY, 1971.

[7] Nirav Dave, Arvind, and Michael Pellauer. Scheduling as Rule Composition. In Proceedings of Formal Methods and Models for Codesign (MEMOCODE), Nice, France, 2007.

[8] Nirav Dave, Man Cheuk Ng, Michael Pellauer, and Arvind. A design flow based on modular refinement. In Formal Methods and Models for Co-Design, 2010. MEMOCODE '10. 8th IEEE/ACM International Conference on, June 2010.

[9] Kermin Fleming, Chun-Chieh Lin, Nirav Dave, Gopal Raghavan, Jamey Hicks, and Arvind. H.264 decoder: A case study in multiple design points. In In Proceedings of Formal Methods and Models for Codesign (MEMOCODE 2008), Anaheim, CA, 2008.

[10] Vijay Ganesh and David L. Dill. A Decision Procedure for Bit-Vectors and Arrays. In 19th International Conference on Computer Aided Verification (CAV-07), pages 519-531, 2007.

[11] James C. Hoe and Arvind. Operation-Centric Hardware Description and Synthesis. IEEE TRANSACTIONS on Computer-Aided Design of Integrated Circuits and Systems, 23(9), September 2004.

[12] Sava Krstić, Robert B. Jones, and John O'Leary. Mothers of pipelines. Electron. Notes Theor. Comput. Sci., 174:7-22, June 2007.

[13] S. Lahiri, S. Seshia, and R. Bryant. Modeling and verification of outof-order microprocessors in UCLID. In FMCAD' 02 , volume 2517 of LNCS, pages 142-159. Springer-Verlag, November 2002.

[14] P. Manolios. A compositional theory of refinement for branching time. In CHARME 2003, volume 2860 of Lecture Notes in Computer Science, pages 304-318. Springer, 2003.

[15] Kenneth L. McMillan. Verification of an implementation of tomasulo's algorithm by compositional model checking. In Proceedings of the 10th International Conference on Computer Aided Verification, CAV '98, pages 110-121, London, UK, 1998.

[16] K. S. Namjoshi. A simple characterization of stuttering bisimulation. In FSTTCS'97, volume 1346 of Lecture Notes in Computer Science, pages 284-296. Springer, 1997.

[17] David A. Patterson and John L. Hennessy. Computer Organization \& Design: The Hardware/Software Interface, Second Edition. Morgan Kaufmann, 1997.

[18] Dominic Richards and David Lester. A monadic approach to automated reasoning for bluespec systemverilog. Innovations in Systems and Software Engineering, pages 1-11, 2011.

[19] Gaurav Singh and Sandeep Shukla. Verifying compiler based refinement of bluespec specifications using the spin model checker. In Model Checking Software, volume 5156 of Lecture Notes in Computer Science, pages 250-269. Springer Berlin / Heidelberg, 2008.

[20] P.J. Windley. Formal modeling and verification of microprocessors. Computers, IEEE Transactions on, 44(1):54 -72, jan 1995. 